\title{
PROGRESS IN HIGH S/N SPECTROSCOPY AND ITS IMPORTANCE FOR STELLAR MODELLING
}

\author{
P. E. NISSEN \\ Institute of Physics and Astronomy, University of Århus, DK-8000 Århus \\ C, Denmark
}

\begin{abstract}
Recent advances in high S/N spectroscopy are reviewed with particular emphasis on new data for the abundances of chemical elements of importance for the modelling of stellar interiors.

It seems well established that young, nearby B-type stars have abundances of CNO elements that are about a factor of two lower than in the Sun except for a small fraction of nitrogen-rich stars. The existence of such stars among main sequence stars remains to be explained.

Among normal A-type stars (excluding $A_{m}$ and $A_{p}$ stars) large deviations from solar abundance ratios occur with interesting anticorrelations of the abundances of $\mathrm{C}$ and $\mathrm{Si}$. This suggests that diffusion processes and/or gas-dust separation of the chemical elements in the protostellar cloud play an important rôle.

For $F$ and $G$ main sequence stars the atmospheric abundance ratios of elements heavier than boron probably represent the original interior composition. For metal-poor stars the abundance ratios are non-solar, and overabundances of oxygen and other $\alpha$-elements have important effects on stellar opacities and energy generation.

The observed abundances of $\mathrm{Li}$ and $\mathrm{Be}$ in $\mathrm{F}$ and $\mathrm{G}$ stars show a complicated behaviour as a function of $T_{e f f}, g,[\mathrm{Fe} / \mathrm{H}]$, and age partly due to depletion of these elements by $(p, \alpha)$ reactions. This provides interesting information on stellar structure, e.g. the depth of the convection zone and rotational mixing. At the same time it is very important that the depletion of ${ }^{6} \mathrm{Li},{ }^{7} \mathrm{Li}$ and ${ }^{9} \mathrm{Be}$ can be modelled so that reliable primordial abundances can be derived.
\end{abstract}

\section{INTRODUCTION}

The advent of new detectors and efficient spectrographs has led to remarkable improvements in observations of stellar spectra at high $\mathrm{S} / \mathrm{N}$ and high resolution. With 4 meter class telescopes it is now possible to obtain spectra of 12th magnitude stars with a resolution of $R \simeq 50.000$ and $S / N \simeq 200$ for exposure times of a few hours, and brighter stars $(V<9.0)$ may be observed with $R \geq 100.000$ and $S / N \simeq 500$. This has led to important new knowledge about stellar properties like:

i) rotation and macroturbulence, 
ii) magnetic fields, star spots and activity,

iii) pulsations and oscillations,

iv) convection, granulation and velocity fields,

v) effective temperatures, gravities and chemical composition.

The first three topics are discussed by other speakers at this colloquium and will not be dealt with in the present review. Studies of convection, granulation and stellar velocity fields will be briefly summarized in the next section, but the main part of the review will deal with new abundance data for various classes of normal main sequence stars and the impact of these data on stellar modelling. As a general theme it is discussed if the observed surface abundances represent the interior composition of the star at zero age.

\section{STELLAR CONVECTION AND GRANULATION}

Spectral signatures of stellar convection and granulation can be observed as asymmetries in photospheric absorption lines, usually described by the line bisectors. Recent work dealing with observations and interpretation of bisectors has been reviewed by Gray (1990) and Dravins (1990a). In general, solar-type stars have $C$ shaped bisectors, that can be explained by numerical, three-dimensional hydrodynamical models of stellar surface convection (Dravins \& Nordlund 1990). It is remarkable that these models are free from classical ad hoc parameters like mixing length, micro- and macro-turbulence.

Based on a survey of bisectors in late $A$ to late $G$ type stars of all luminosity classes Gray \& Nagel (1989) have shown that a granulation boundary exists in the HR diagram. The boundary runs from F0V to G1Ib. On the cool side of the boundary the spectral line bisectors have $C$ shapes corresponding to a granulation pattern as known from the Sun with characteristic velocities of a few $\mathrm{km} \mathrm{s}^{-1}$. On the hot side of the boundary the bisectors are inverted having $\supset$ shapes. Similar bisectors have been observed in early-type stars like Sirius (A1 V; Dravins 1987) and $\tau$ Sco (B0 V; Smith \& Karp 1978, 1979). As discussed by Dravins (1990b) these bisectors suggest that the stars are covered by small granules corresponding to upwelling currents in "geysers" with velocities of perhaps $10-20 \mathrm{~km} \mathrm{~s}^{-1}$ balanced by a slower downdraft in more extended areas. The source of these strong velocity fields remains an open question, but it is interesting that non-LTE analyses of stellar equivalent widths in B and A type stars also suggest that strong velocity fields exist in their photospheres. Thus, Kilian (1992) and Gies \& Lambert (1992) derive microturbulence velocities of $5-10 \mathrm{~km} \mathrm{~s}^{-1}$ in B0-B3 V stars, and Lemke (1989) finds velocities of $2-3 \mathrm{~km} \mathrm{~s}^{-1}$ in $\mathrm{A} 0-\mathrm{A} 2 \mathrm{~V}$ stars.

In addition to new information about stellar convection obtained from the study of spectral line bisectors, such data also provides important checks on inhomogeneous models of stellar atmospheres based on hydrodynamical codes (e.g. Nordlund \& Dravins 1990). The construction of such models for a large range of the basic atmospheric parameters, $T_{\text {eff }}, g$, and $[\mathrm{Fe} / \mathrm{H}]$, would be important in order to improve the accuracy of stellar abundance determinations. Thus, the puzzling differences between oxygen abundances of metal-poor stars derived from the infrared OI triplet at $7774 \AA$ and the forbidden [OI] line at 
$6300 \AA$ may well be due to the neglect of inhomogeneities in the models used to analyze the observed equivalent widths (Kiselman 1991; Tomkin et al. 1992).

\section{THE CHEMICAL COMPOSITION OF MAIN SEQUENCE STARS}

In this section we discuss recent work on the abundances of some key elements in various classes of normal main sequence stars. These works are based on high resolution spectra observed with high S/N and analyzed by the aid of non-LTE model atmosphere computations. At the same time the works are characterized by including a relative large number of stars so that statistical significant results are obtained.

CNO and Si abundances in early B-type stars

Two important studies of CNO abundances in early B main sequence stars have recently appeared. The first one by Gies and Lambert (1992) includes 34 'northern' stars mostly of spectral types B0-B3 and luminosity classes V and IV. The stars were observed with the McDonald $2.1 \mathrm{~m}$ telescope with a resolution of $R \simeq 20.000$ and a $S / N \simeq 300$ in four bands of about $100 \AA$ in the spectral region $4200-5200 \AA$. Equivalent widths of about 70 absorption lines - some as faint as $5 \mathrm{~m} \AA$ - were measured for each star. Effective temperatures of the stars were determined from the Strömgren Balmer discontinuity index $\left[c_{1}\right]$ and surface gravities from the profile of the $H_{\gamma}$ line. LTE abundances were first derived using the line blanketed model atmospheres of Kurucz (1979) and then compared with non-LTE abundances based on the equivalent tables of Eber \& Butler (1988) (CII), Becker \& Butler (1988a) (NII) and Becker \& Butler (1988b) (OII). The two sets of abundances agree well with maximum differences of about 0.20 dex.

The second study of abundances of CNO elements in early B-type stars has been carried out by Kilian (1992), and is based on spectra of 20 'southern' B0B3 main sequence stars obtained with the ESO $3.6 \mathrm{~m}$ telescope and its CASPEC spectrograph with a resolution of 20.000 and a $S / N \simeq 150$. The whole spectral region 4000-5000 $\AA$ was observed and equivalent widths of up to 250 lines per star are published by Kilian \& Nissen (1989). Atomic data including oscillator strengths for the lines are given by Kilian, Montenbruck \& Nissen (1991). Effective temperatures of the stars have been determined from a non-LTE analysis of Si II, Si III and Si IV lines and gravities are determined from the profiles of $H_{\gamma}$ lines (Kilian et al. 1991). The derived abundances of the CNO elements are based on the non-LTE computations of Eber, Becker \& Butler referred to above. The errors of the abundances are estimated to be about \pm 0.10 dex.

The B stars analyzed by Gies \& Lambert and Kilian lie within $1 \mathrm{kpc}$ from the Sun and include several stars in the Orion and Sco-Cen associations. In order to avoid severe blending of spectral lines the sample has been restricted to stars with projected rotational velocities $V \sin i<80 \mathrm{~km} \mathrm{~s}^{-1}$. Five stars are in common between the two studies and their derived $\mathrm{CNO}$ abundances agree within a rms deviation of about 0.15 dex.

Fig. 1 shows the frequency of stars as a function of $[\mathrm{X} / \mathrm{H}] \equiv \log \left(N_{X} / N_{H}\right)_{s t a r}-$ $\log \left(N_{X} / N_{H}\right)_{S u n}$ for $\mathrm{X}=\mathrm{C}, \mathrm{N}$ and $\mathrm{O}$. As seen the results of the two works described above agree very well. The widths of the histograms of $\mathrm{C}$ and $\mathrm{O}$ abun- 

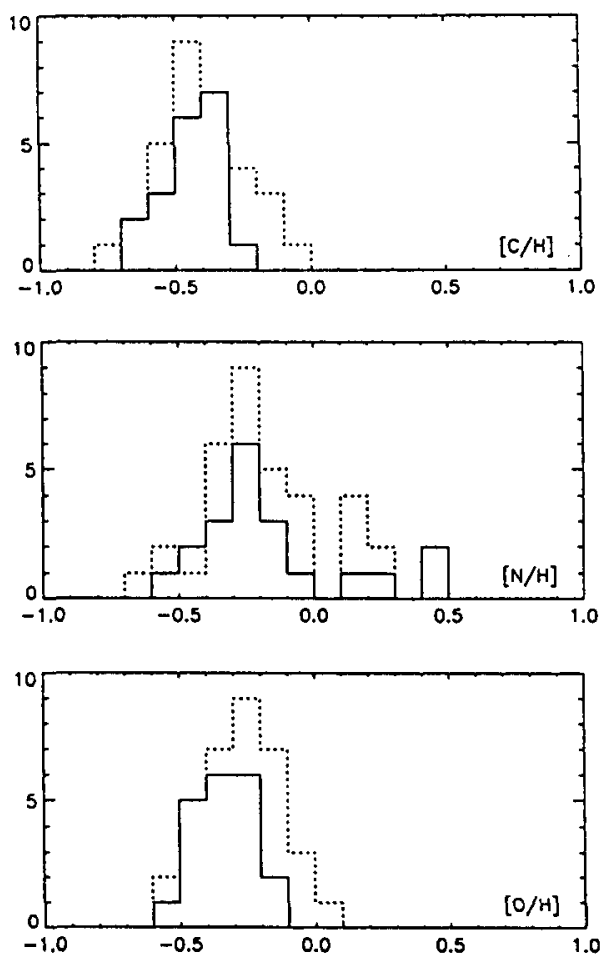

FIGURE I Number of B0-B3 main sequence stars versus the logarithmic abundances of $\mathrm{C}, \mathrm{N}$ and $\mathrm{O}$ relative to the solar abundances with data taken from Gies \& Lambert (1992) (dotted line) and Kilian (1992) (solid line)

dances are consistent with the errors of the abundance determinations, whereas the histograms of the $\mathrm{N}$ abundances are much broader with a tail of positive values of $[\mathrm{N} / \mathrm{H}]$, which cannot be explained by errors in the abundance analyses.

Excluding for a moment the nitrogen-rich stars, it is seen that the mean values of the abundances of $\mathrm{C}, \mathrm{N}$ and $\mathrm{O}$ are about a factor of two lower than the solar abundances, i.e. $[\mathrm{X} / \mathrm{H}] \simeq-0.3$. This surprising result can hardly be due to systematic errors in the model atmosphere analysis of the observed equivalent widths or in the atomic data - the calculated oscillator strengths of the lines are estimated to be accurate to about $10 \%$. It means that nearly all nearby $\mathrm{B}$ main sequence stars have metallicities of $Z \simeq 0.01$ instead of the solar value, $Z_{\odot}=0.02$. It remains to be investigated if this low metallicity is also representative for the stellar interiors or if the surface layers have been depleted in CNO by e.g. selective mass loss. The fact that the local HII regions are also deficient in oxygen by a factor of two relative to the Sun (Peimbert 
1987) suggests, however, that the low abundance of CNO elements is a general phenomenon of gas and young stars in the solar neighborhood of the Galaxy. Information on other heavy elements is scarce. Note, however, that Kilian (1992) also derives a mean value of the $\mathrm{Si}$ abundance 0.3 dex below the solar value.

In stellar modelling it is normally assumed that the metallicity of nearby $B$ stars is higher than or equal to the solar value, $Z_{\odot}=0.02$, because one expects the metallicity of the interstellar gas to increase as a function of time due to chemical evolution in the Galaxy. Recent, accurate data on abundances and ages of $\mathrm{F}$ dwarfs (Edvardsson et al. 1992) shows, however, that there is no smooth age-metallicity relation for the galactic disk. At a given time and galactocentric distance the variation in the abundance of heavy elements amounts to almost a factor of two. Such variations may be related to infall of relatively metal-poor gas that triggers star formation before mixing in the galactic disk. In any case, model builders should be open for the possibility that the interior composition of young, nearby stars is characterized by a metallicity of $Z \simeq 0.01$ with the interesting consequences this may have for stellar opacities, energy generation, mass loss etc. As an example we refer to the recent work by Moskalik \& Dziembowski (1992) on the origin of $\beta$ Cephei pulsation. Using the new opacities of Iglesias \& Rogers (1991a,b) they suggest that the driving of the pulsations is caused by the $\kappa$-mechanism acting in a zone with temperatures near $2 \cdot 10^{5} \mathrm{~K}$ where there is a bump in opacity. However, as shown in their Fig. 1 this mechanism works only if $Z>0.02$. In this connection we note that several $\beta$ Cephei stars are included in the abundance studies of Gies \& Lambert and Kilian and have $\mathrm{C}$, $\mathrm{N}, \mathrm{O}$, and $\mathrm{Si}$ abundances, corresponding to $Z \simeq 0.01$.

The existence of nitrogen-rich stars is another puzzling problem. Lyubimkov (1991) has presented evidence that the nitrogen abundance of $B$ main sequence stars is correlated with stellar age and has suggested that mixing exists between the outer layers and the interior leading to a gradual transfer of CNO-cycle products from the stellar interior to the atmosphere. Gies \& Lambert, however, find no significant correlation between stellar age and nitrogen abundance, and as discussed by Lambert (1992) other parameters than mass, age and initial composition seem to control the mixing of CNO-cycled material into the atmosphere. Rotation is a good candidate. Although the nitrogen-rich stars observed have low projected rotational velocities they may be fast rotators seen close to pole on. In any case, it is a surprise that mixing can occur in main sequence stars and a detailed explanation remains to be presented.

\section{$\mathrm{C}, \mathrm{Si}$ and $\mathrm{Fe}$ abundances in early A-type stars}

A thorough study of the chemical composition of early A-type stars has been conducted by the stellar atmospheric group in Kiel (Holweger et al. 1986a,b). The spectra analyzed have primarily been obtained with the ESO Coudé Echelle Spectrometer at a resolution of 50.000. The sample is restricted to $17 \mathrm{~B} 9.5 \mathrm{~V}$ A2V stars classified as normal in the Bright Star Catalogue (Hoffleit \& Jaschek 1982). Detailed non-LTE model atmosphere analyses of the equivalent width data have been presented by Gigas $(1986,1988)$ and Lemke $(1989,1990)$, and have led to the discovery of surprisingly large variations from star to star in the abundance of iron peak elements accompanied by parallel variations of $\alpha$ elements ( $\mathrm{Si}$ and $\mathrm{Ca}$ ). Thus, Sirius has twice the solar abundance of these ele- 
ments, whereas Vega is underabundant with about a factor of four with respect to the Sun. As recently discussed by Holweger (1992) the carbon abundance is anticorrelated with the $\mathrm{Si}$ and $\mathrm{Fe}$ abundance in the sense that [C/Si] is high when $[\mathrm{Si} / \mathrm{H}]$ or $[\mathrm{Fe} / \mathrm{H}]$ is low and vice versa. Holweger points out that $\mathrm{C}$ differs from these elements by having a low condensation temperature, $T_{c}=80 \mathrm{~K}$, while the other elements have $T_{c}>1200 \mathrm{~K}$. Carbon is therefore less depleted in the interstellar gas than $\mathrm{Si}$ and $\mathrm{Fe}$. Hence, the large variations in the $\mathrm{Si}$ and $\mathrm{Fe}$ abundances as well as the anticorrelation of $[\mathrm{C} / \mathrm{Si}]$ with $[\mathrm{Si} / \mathrm{H}]$ could be due to a variable gas/dust ratio in the interstellar matter from which the stars were formed. This would probably mean that the initial interior composition of these A-type stars is rather different from the solar composition with interesting consequences for the stellar models. On the other hand it may also be that the peculiar composition of the A-type stars has been created during later accretion of gas and dust in variable ratios. Then the atmospheric composition is different from the initial composition of the star. The same would be the case if the peculiar composition is due to diffusion processes.

The large abundance variations (almost a factor of 10 in metallicity) among normal A-type stars is a very surprising result. In young $F$ or $B$ stars the variation in metallicity is less than a factor of 2 . Also there is no sign of an anticorrelation between the abundance of $\mathrm{C}$ and $\mathrm{Si}$ in $\mathrm{B}$ and $\mathrm{F}$ stars. Why are the early A-type stars behaving in a special way?

Oxygen, $\alpha$-element and iron abundances in $\mathrm{F}$ and $\mathrm{G}$ stars

For $F$ and $G$ main sequence stars the observed abundances of elements heavier than boron probably represent the initial stellar composition. There are two observational arguments for this statement. First, the solar photospheric composition agrees very well with the composition of meteorites (Anders \& Grevesse 1989). In particular, we note that the recent revision of the iron abundance in the solar photosphere (Biémont et al. 1991; Holweger et al. 1991) has led to a nearly perfect agreement with the meteoritic iron abundance. Second, the abundance of a given element in open cluster stars seems constant in the effective temperature range $5500<T_{\text {eff }}<7000 \mathrm{~K}$. Thus, Cayrel et al. (1985) and Boesgaard \& Friel (1991) find [Fe/H] to be constant within \pm 0.05 dex for the Hyades. A similar small upper limit on the intrinsic abundance dispersion of $\mathrm{C}$ is found by Friel \& Boesgaard (1991) for several young open clusters, whereas Garcia Lopez et al. (1992) find the oxygen abundance in the Hyades stars to be constant within \pm 0.10 dex for $5800<T_{\text {eff }}<7400 \mathrm{~K}$. If atomic diffusion or hydrodynamical processes played a rôle in modifying the surface composition for elements heavier than boron one would expect to observe some $T_{\text {eff }}$ dependence of cluster abundances of these elements like in the case of $\mathrm{Li}$ and $\mathrm{Be}$.

Assuming then that $F$ and $G$ stars reflect the composition of pre-stellar clouds they can be used for mapping the chemical composition of the Galaxy as a function of age and galactocentric distance. Furthermore, trends and scatter of various abundance ratios as a function of metallicity provide important information about nucleosynthesis in stars and supernovae. At the same time it is essential to know the number ratios of the most abundant elements for stars with different metallicities in order to construct reliable models to be used for e.g. age determinations. In the following some interesting new results concerning $\mathrm{O}$ 


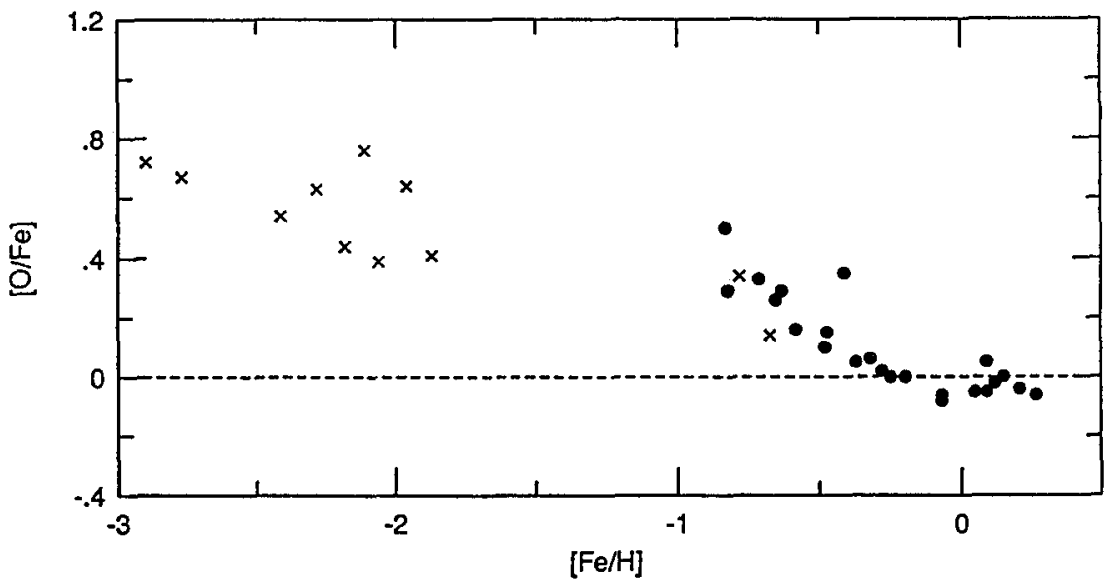

FIGURE II The oxygen-to-iron ratio as a function of metallicity in $\mathrm{F}$ and $\mathrm{G}$ dwarf stars. •, refers to oxygen abundance determinations based on equivalent widths of the faint, forbidden oxygen line at $6300 \AA$ by Nissen $\&$ Edvardsson (1992). X, refers to oxygen abundances derived from $\mathrm{OH}$ lines in the near ultraviolet by Gilmore et al. (1992)

and other $\alpha$-element abundances as a function of $\mathrm{Fe} / \mathrm{H}$ are discussed.

Existing data on the $\mathrm{O}$-Fe relation in the galactic disk and halo are in severe disagreement. From analyses of the OI triplet at $7774 \AA$ in $F$ and $G$ dwarfs a steep relation, $[\mathrm{O} / \mathrm{Fe}] \simeq-0.5[\mathrm{Fe} / \mathrm{H}]$, is found both for disk stars (Andersen et al. 1988) and halo stars (Abia \& Rebolo 1989), whereas the forbidden lines at 6300 and $6363 \AA$ in $\mathrm{K}$ giants lead to a shallower relation for disk stars (Barbuy $\&$ Erdelyi-Mendes 1989 ) and a constant value, $[\mathrm{O} / \mathrm{Fe}] \simeq 0.35$, for halo stars (Barbuy 1988, Sneden et al. 1991). The discrepancy amounts to about 1.0 dex in $[\mathrm{O} / \mathrm{Fe}]$ for the most metal-deficient halo stars. Recent observations of the [OI] line in metal-poor dwarf stars (Spite \& Spite 1991, Spiesman \& Wallerstein 1991) show that the 0 abundances derived from the triplet lines are 0.3 to 0.5 dex too high. Apparently, this cannot be explained in terms of non-LTE effects (Kiselman 1991, Tomkin et al. 1992). Instead, the discrepancy may be due to the neglect of thermal inhomogeneities in the model atmospheres, because the population of the high excitational energy levels corresponding to the triplet lines is very sensitive to temperature. In any case, it seems that the $O$ abundances derived from the triplet are not reliable. In Fig. 2 we therefore show some new results based on accurate observations of the faint [OI] line in F and $G$ dwarfs by Nissen \& Edvardsson (1992) as well as oxygen abundances derived from high resolution observations of $\mathrm{OH}$ lines in the near ultraviolet region, $3138-3155 \AA$ by Gilmore et al. (1992). As seen the $0 / F e$ ratio is close to the solar ratio for the metallicity range $-0.3<[\mathrm{Fe} / \mathrm{H}]<+0.3$. Below $[\mathrm{Fe} / \mathrm{H}]=-0.3$ the $0 / \mathrm{Fe}$ ratio increases steeply and reaches $[0 / \mathrm{Fe}] \simeq 0.4$ at $[\mathrm{Fe} / \mathrm{H}] \simeq-0.8$. The halo stars in Fig. 2 have a mean ratio of $[0 / \mathrm{Fe}] \simeq 0.5$ i.e. three times the solar $\mathrm{O} / \mathrm{Fe}$ ratio. It is, however, unclear from the data whether the $\mathrm{O} / \mathrm{Fe}$ ratio in halo stars 


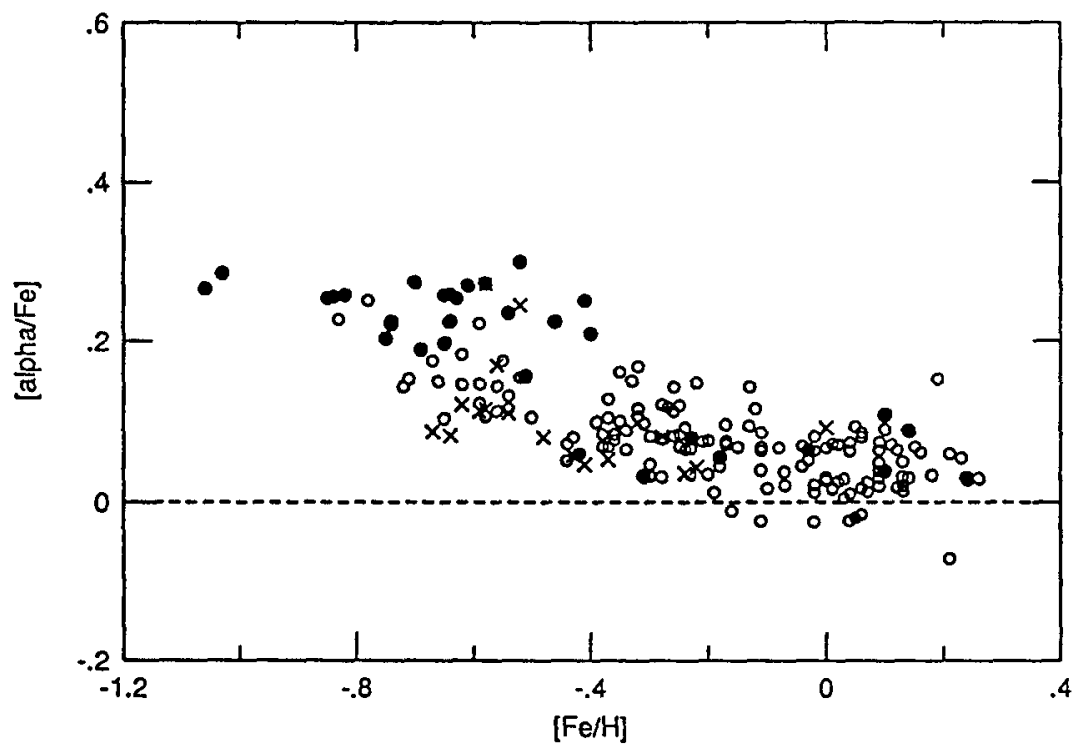

FIGURE III The abundance ratio between $\alpha$-elements and iron as a function of metallicity for $\mathrm{F}$ and $\mathrm{G}$ stars with different mean galactocentric dis-

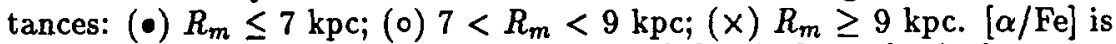
defined as the mean value of $[\mathrm{Mg} / \mathrm{Fe}],[\mathrm{Si} / \mathrm{Fe}],[\mathrm{Ca} / \mathrm{Fe}]$ and $[\mathrm{Ti} / \mathrm{Fe}]$

is constant or depends on metallicity. Furthermore, there may be a significant cosmic dispersion in $0 / \mathrm{Fe}$ at a given $[\mathrm{Fe} / \mathrm{H}]$. Such a dispersion is indeed found for other $\alpha$-elements, as discussed in the next section.

A survey of the chemical composition of $189 \mathrm{~F}$ and $\mathrm{G}$ dwarf stars in the galactic disk has recently been completed by Edvardsson et al. (1992). The survey is based on high resolution observations in selected spectral bands with the McDonald $2.7 \mathrm{~m}$ and the ESO $1.4 \mathrm{~m}$ CAT telescopes. The stars were selected without kinematical bias from the big catalogues of $u v b y-\beta$ photometry (Olsen 1983 ) and cover the metallicity range $-1.0<[\mathrm{Fe} / \mathrm{H}]<+0.3$ uniformly. Accurate radial velocities, proper motions and distances are available for the stars so that galactic orbits and mean galactocentric distances $R_{m}$ can be calculated. As shown by Grenon (1987), $R_{m}$ is a good estimate of the galactocentric distance at which the star was formed.

The equivalent widths measured were analyzed by the aid of new, fully lineblanketed model atmospheres. $T_{\text {eff }}$ and $g$ were determined from the $u v b y-\beta$ photometry. The excitation and ionization balance of iron lines suggest that these two parameters are accurate to $\pm 100 \mathrm{~K}$ and \pm 0.15 dex, respectively. The derived abundances assume LTE population of the atomic levels, but non-LTE calculations were carried out to ensure that this simplifying assumption introduces negligible errors.

The analysis of the data has led to the determination of abundances of $O$, $\mathrm{Na}, \mathrm{Mg}, \mathrm{Al}, \mathrm{Si}, \mathrm{Ca}, \mathrm{Ti}, \mathrm{Fe}, \mathrm{Ni}, \mathrm{Y}, \mathrm{Zr}, \mathrm{Ba}$ and $\mathrm{Nd}$ relative to the solar abundances. 
Interesting information on the trends and scatter of various abundance ratios and correlations with stellar age and kinematical parameters are obtained. Here we only comment on the results for $\mathrm{Mg}, \mathrm{Si}, \mathrm{Ca}$ and $\mathrm{Ti}$, which all behave in a similar way as oxygen. The mean abundance of these four elements is plotted in Fig. 3 as a function of $[\mathrm{Fe} / \mathrm{H}]$ with different symbols for stars born in the inner parts of the Galaxy, in the solar 'cylinder' and in the outer parts. For $[\mathrm{Fe} / \mathrm{H}]>-0.4$ the three groups are well mixed with an average value $[\alpha / \mathrm{Fe}]=$ 0.05. Apparently, this small offset from the solar ratio cannot be explained as due to errors in the analysis. For $[\mathrm{Fe} / \mathrm{H}]<-0.4$ the three groups separate. At a given $[\mathrm{Fe} / \mathrm{H}]$ stars born in the inner part of the Galaxy tend to have higher values of $[\alpha / \mathrm{Fe}]$ than stars born in the outer part. This behaviour can be explained by assuming that the rate of chemical evolution in the galactic disk is a decreasing function of galactocentric distance (see discussion in Edvardsson et al. 1992). As a direct consequence for stellar modelling we conclude that it is not sufficient to characterize the interior composition by only two independent parameters, say $X$ and $Z$. One needs at least one additional parameter specifying the $\alpha / \mathrm{Fe}$ ratio, where $\alpha$ refers to elements synthesized by the $\alpha$-process in massive, short-lived stars exploding as Type II supernovae ( $\mathrm{O}, \mathrm{Ne}, \mathrm{Mg}, \mathrm{S}, \mathrm{Si}$ etc.).

The effect of variations in the $\mathrm{O} / \mathrm{Fe}$ ratio on models for metal-poor stars has been investigated in detail by VandenBerg (1992). At $[\mathrm{Fe} / \mathrm{H}] \simeq-2.0$ an increase of $[0 / \mathrm{Fe}]$ from 0.0 to 0.75 leads to a decrease of globular cluster ages derived from turnoff luminosities by 2.2 Gyr primarily due to changes in the H-burning nucleosynthesis in the CNO cycle. The opacity effect arising from enhanced oxygen are considerably less important than its nucleosynthesis effects at this low metallicity. At higher metallicities, say $[\mathrm{Fe} / \mathrm{H}]=-0.5$, one would expect that the opacity effect plays a larger rôle especially if the enhancement of the other $\alpha$-elements ( $\mathrm{Ne}, \mathrm{Mg}, \mathrm{Si}, \mathrm{S}$, etc.) is taken into account. In particular, it would be interesting to see if the high ages $(15 \mathrm{Gyr})$ of thick disk stars with $[\mathrm{Fe} / \mathrm{H}] \simeq-0.5$ determined by Edvardsson et al. (1992) will be reduced when isochrones corresponding to enhanced $\alpha$-element abundances are applied.

\section{$\mathrm{Li}, \mathrm{Be}$ and $\mathrm{B}$ abundances in $\mathrm{F}$ and $\mathrm{G}$ stars}

The abundances of light elements ( $\mathrm{Li}, \mathrm{Be}$ and $\mathrm{B}$ ) in late type stars depend on nuclear processes in at least three different environments: (i) nucleosynthesis in the Big Bang phase of the Universe, (ii) interaction between cosmic rays and interstellar gas, $(i i i)(p, \alpha)$ reactions in stellar interiors. Hence, by studying the abundances of these light elements as a function of stellar parameters like $T_{\text {eff }}$, $g,[\mathrm{Fe} / \mathrm{H}]$, age, mass, and rotation we have the possibility to obtain information about the physical conditions in the early Universe, in the Galaxy, and in the stars. It is, however, difficult to disentangle the various processes leading to the observed surface abundances of $\mathrm{Li}, \mathrm{Be}$ and $\mathrm{B}$ in a given star.

Observations of $\mathrm{Li}$ in Pop. I and II stars have recently been reviewed by Spite (1991), Molaro(1991), Balachandran (1991) and Soderblom (1991). Most notable are the constancy of $\mathrm{Li}$ abundance among halo stars with $T_{\text {eff }}>5500 \mathrm{~K}$, the dip in $\mathrm{Li}$ abundance in open cluster stars at $T_{\text {eff }} \simeq 6600 \mathrm{~K}$, and the complicated dependence of $\mathrm{Li}$ abundances on age and $[\mathrm{Fe} / \mathrm{H}]$ for $T_{\text {eff }}<6000 \mathrm{~K}$. From theoretical studies it is well known that the temperature at the bottom of the convection zone is not high enough to explain the observed degree of $\mathrm{Li}$ 
depletion. Additional mixing caused by overshooting, gravity waves, mass loss, rotation or diffusion is needed. For a recent discussion of these mechanisms the reader is referred to Schatzman (1991) and Michaud \& Richer (1991).

A recent accurate survey of lithium in 81 main-sequence stars with $5800<$ $T_{\text {eff }}<6700$ by Lambert, Heath \& Edvardsson (1991) has augmented our knowledge to $\mathrm{Li}$ abundances in disk stars considerably. A novel result of this survey is that, for the large majority of old disk dwarfs too low in mass to belong to the Li-gap, Li is only mildly depleted. In a small fraction (about $10 \%$ ) of the stars $\mathrm{Li}$ is, however, strongly depleted by a factor of 10 or more. Apparently, stars of intermediate $\mathrm{Li}$ abundance do not exist. This suggests that $\mathrm{Li}$ depletion in stars is influenced by more than the standard stellar variables of mass, age and metallicity. It is of great interest to find out which additional parameter (e.g. rotation, mass loss or binarity) plays a decisive rôle for the degree of $\mathrm{Li}$ depletion.

Beryllium abundances in disk stars have been reviewed by Boesgaard (1991). For field stars a dichotomy seems to exist. Most stars have the same Be abundance as the Sun $(\log (\mathrm{Be} / \mathrm{H})=-10.85)$, but some $\mathrm{F}$ dwarfs in the Li-gab are markedly deficient in Be. The Be-deficient stars all have severe Li deficiencies, but many $\mathrm{Li}$-deficient stars have solar Be abundances. This makes sense because Be destruction by $(p, \alpha)$ reactions requires a higher temperature than $\mathrm{Li}$ destruction. Again, other parameters than mass, age and metallicity seem to be decisive for the occurrence of Be depletion.

Recently, beryllium and boron abundances have been determined for very metal-poor stars. Gilmore, Edvardsson \& Nissen (1991) succeeded to detect the Be II resonance doublet at $3131 \AA$ in $H D 140283([\mathrm{Fe} / \mathrm{H}] \simeq-2.7)$ using the Anglo-Australian Telescope and the UCL Echelle Spectrograph with a resolution of $R \simeq 60.000$. Later Ryan et al. (1992) and Gilmore et al. (1992) have determined $\mathrm{Be}$ abundances in other metal-poor stars. Furthermore, Duncan, Lambert \& Lemke (1992) have derived Boron abundances in three metal-poor stars from observations of the BI resonance lines at $2497 \AA$ observed with the Goddard High Resolution Spectrograph of the Hubble Space Telescope. The data on Be and B is shown in Fig. IV. As seen both elements have abundances proportional to the abundance of iron. The scatter in the relations can be explained by errors in the abundance determinations, and the linear dependence of $\mathrm{Be}$ and $\mathrm{B}$ on $\mathrm{Fe}$ (and $\mathrm{O}$ ) as well as the constant $\mathrm{B} / \mathrm{Be}$ ratio of about 10 are compatible with cosmic ray spallation production of Be and $B$, although current models for this production have to be modified (Gilmore et al. 1992, Duncan et al. 1992). Hence, there is no evidence for primordial Big Bang production of $\mathrm{Be}$ and $\mathrm{B}$. Neither, there is evidence for depletion of $\mathrm{Be}$ and $\mathrm{B}$ in these metalpoor stars, which have $5500<T_{\text {eff }}<6200 \mathrm{~K}$. Lithium depletion in metalpoor halo stars is, however, a controversial problem. As shown by Deliyannis et al. (1990) classical, non-rotating models predict a negligible degree of ${ }^{7} \mathrm{Li}$ depletion in metal-poor stars with $T_{\text {eff }}>5500 \mathrm{~K}$. Models of halo stars with rotation predict, however, a depletion factor between 5 and 10 depending on the initial angular momentum of the star (Pinsonneault et al. 1992). If correct, the primordial Big Bang lithium abundance may be as high $\mathrm{Li} / \mathrm{H} \simeq 10^{-9}$ instead of the canonical value of $1.510^{-10}$. Observations of the ${ }^{6} \mathrm{Li} /{ }^{7} \mathrm{Li}$ ratio in metal-poor stars would be helpful in solving this crucial problem. Pilachowski et al. (1989) 


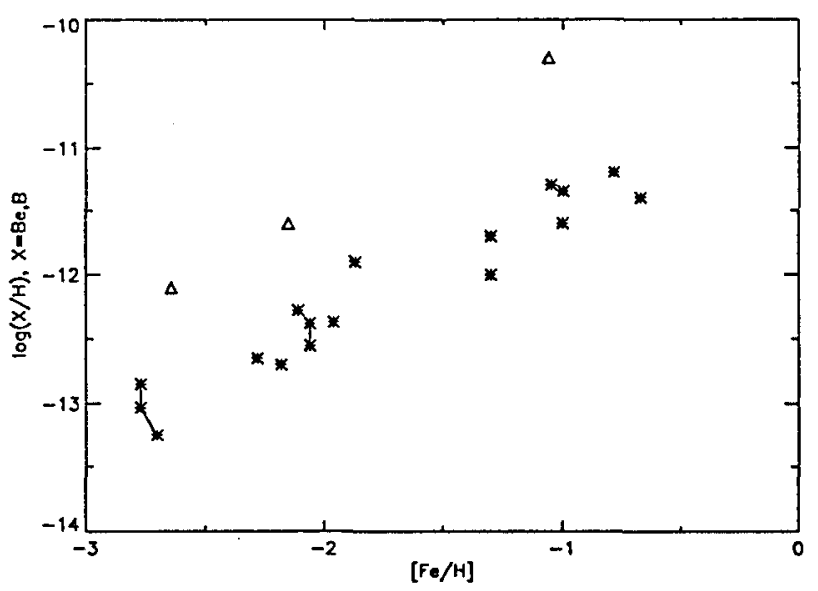

FIGURE IV Beryllium and boron abundances in metal-poor stars as a function of metallicity. *, Be abundances from Rebolo et al. (1988), Ryan et al. (1992) and Gilmore et al. (1992). $\triangle, B$ abundances from Duncan et al. (1992). Points referring to different observations of the same star are connected with straight lines

have searched for ${ }^{6} \mathrm{Li}$ in $\mathrm{HD} 84937\left(T_{\text {eff }} \simeq 6200 \mathrm{~K},[\mathrm{Fe} / \mathrm{H}] \simeq-2.4\right)$ by observing the profile and wavelength of the $\mathrm{Li}$ doublet at $6707 \AA$ and have obtained an upper limit ${ }^{6} \mathrm{Li} /{ }^{7} \mathrm{Li}<0.10$. Recently, Smith, Lambert \& Nissen (1992) have reported a detection of ${ }^{6} \mathrm{Li}$ in the same star at a level of ${ }^{6} \mathrm{Li} /{ }^{7} \mathrm{Li} \simeq 0.05$ based on very high $\mathrm{S} / \mathrm{N}$ observations of the doublet with the McDonald $2.7 \mathrm{~m}$ telescope. If confirmed, this would support the standard models, because the fragile ${ }^{6} \mathrm{Li}$ isotope is completely destroyed in models with rotational mixing (Deliyannis 1990).

\section{CONCLUSIONS}

As emphasized in this review and as discussed in more detail by other authors in these proceedings high $\mathrm{S} / \mathrm{N}$ spectroscopy provides information on many aspects of stellar modelling. Much new information relating to stellar rotation, convection, magnetic fields and chemical composition has been obtained during the last few years. In particular, we have discussed some recent work on abundances of key elements in main sequence stars. Several novel results have been obtained. CNO abundances in young B stars turn out to be a factor of two lower than in the Sun with the exception of the (unexplained) occurrence of a small fraction of nitrogen-rich stars. Among the A-type stars large (up to a factor of 10) variations of iron-peak and $\alpha$-element abundances are found with interesting anticorrelations of $\mathrm{C}$ and $\mathrm{Si}$. For the $\mathrm{F}$ and $\mathrm{G}$ stars the trends and scatter in various abundance ratios as a function of metallicity have been studied in detail with important consequences for nucleosynthesis and opacity in stellar 
interiors. In addition, observations of ${ }^{6,7} \mathrm{Li}, \mathrm{Be}$ and $\mathrm{B}$ abundances are providing new insight in Big Bang nucleosynthesis, cosmic rays in the early Galaxy and stellar structure. We conclude that the field of high $\mathrm{S} / \mathrm{N}$ spectroscopy is likely to continue to contribute significantly to our knowledge about stellar structure and to other branches of astrophysics as well.

\section{REFERENCES}

Abia, C., Rebolo, R. 1989, ApJ, 347186.

Anders, E., Grevesse, N. 1989, Geochim. Cosmochim. Acta, 53, 197.

Andersen, J., Edvardsson, B., Gustafsson, B., Nissen, P.E. 1988, in Proc. IAU Symp. No. 132, eds. G. Cayrel de Strobel and M. Spite (Dordrecht: Kluwer), p. 485.

Balachandran, S. 1991, Mem.S.A.It., 62, 33.

Barbuy, B. 1988, $A \& A, 191,121$.

Barbuy, B., Erdelyi-Mendes, M. 1989, A\&A, 214, 239.

Becker, S.R., Butler, K. 1988a, $A \& \dot{A} S, 76,331$.

Becker, S.R., Butler, K. 1988b, $A \& A S, 74,211$.

Biémont, E., Baudoux, M., Kurucz, R., Ansbacher, W., Pinnington, E.H. 1991, $A \& A, 249,539$.

Boesgaard, A.M. 1991 in The Formation and Evolution of Star Clusters, Ed. K. Janes, $P A S P C, 13$ (in press).

Boesgaard, A.M., Friel, E.D. 1991, ApJ, 351, 467.

Cayrel, R., Cayrel de Strobel, G., Campbell, B. 1985, A\&A, 146, 249.

Deliyannis, C.P. 1990, Ph.D. dissertation, Yale University.

Deliyannis, C.P., Demarque, P., Kawaler, S.D. 1990. ApJS, 73, 21.

Dravins, D. 1987, $A \& A, 172,211$.

Dravins, D. 1990a, Mem.S.A.It, 61, 513.

Dravins, D. 1990b, $A \& A, 228,218$.

Dravins, D., Nordlund, A. 1990, $A \& A, 228,203$.

Duncan, D.K., Lambert, D.L., Lemke, M. 1992, $A p J$ (submitted).

Eber, F., Butler, K. 1988, A\&A, 202, 153.

Edvardsson, B., Andersen, J., Gustafsson, B., Lambert, D.L., Nissen, P.E., Tomkin, J. 1992 (in preparation).

Friel, E.D., Boesgaard, A.M. 1991, ApJ, 351, 480.

Garcia Lopez, R.J., Rebolo, R., Herrero, A., Beckman, J.E. 1992 (these proceedings).

Gies, D.R., Lambert, D.L. 1992, $A p J, 387,673$.

Gigas, D. 1986, $A \& A, 165,170$. 
Gigas, D. 1988, $A \& A, 192,264$.

Gilmore, G., Edvardsson, B., Nissen, P.E. 1991, ApJ, 378, 17.

Gilmore, G., Gustafsson, B., Edvardsson, B., Nissen, P.E. 1992, Nature, 357, 379.

Gray, D.F. 1990, Mem.S.A.It., 61, 503.

Gray, D.F., Nagel, T. 1989, ApJ, 341, 421.

Grenon, M. 1987, JA\&A, 8, 123.

Hoffleit, D., Jaschek, C. 1982, The Bright Star Catalogue, Yale University Observatory, New Haven.

Holweger, H. 1992, in Atmospheres of Early-Type Stars, Eds. U. Heber \& C.S. Jeffery, Lecture Notes in Physics (Heidelberg: Springer) (in press).

Holweger, H., Bard, A., Kock, A., Kock, M. 1991, A\&A, 249, 545.

Holweger, H., Gigas, D., Steffen, M. 1986a, $A \& A, 155,58$.

Holweger, H., Steffen, M., Gigas, D. 1986b, $A \& A, 163,333$.

Iglesias, C.A., Rogers, F.J. 1991a, $A p J, 371,408$.

Iglesias, C.A., Rogers, F.J. 1991b, ApJ, 371, L73.

Kilian, J. 1992, $A \& A$, (submitted).

Kilian, J., Becker, S.R., Gehren, T., Nissen, P.E. 1991, $A \& A, 244,419$.

Kilian, J., Montenbruck, O., Nissen, P.E. 1991, $A \& A S, 88,101$.

Kilian, J., Nissen, P.E. 1989, A\&AS, 80, 255.

Kiselman, D. 1991, $A \& A, 245$, L9.

Kurucz, R.L. 1979. ApJS, 40, 1.

Lambert, D.L. 1992 in High Resolution Spectroscopy with the VLT, ed. M.-H. Ulrich, ESO Workshop, February 11-13, 1992 (ESO: Garching) (in press).

Lambert, D.L., Heath, J.E., Edvardsson, B. 1991, MNRAS, 253, 610.

Lemke, M. 1989, $A \& A, 225,125$.

Lemke, M. 1990, $A \& A, 240,331$.

Lyubimkov, L.S. 1991 in Evolution of Stars: The Photospheric Abundance Connection, eds. G. Michaud \& A. Tutukov, IAU Symp. 145 (Dordrecht: Kluwer), p. 125.

Michaud, G., Richer, J. 1991, Mem.S.A.It., 62, 151.

Molaro, P. 1991, Mem.S.A.It., 62, 17.

Moskalik, P., Dziembowski, W.A. 1992, A\&A, 265, 65.

Nissen, P.E., Edvardsson, B. 1992, $A \& A$, (in press).

Nordlund, A., Dravins, D. 1990, $A \& A, 228,155$.

Olsen, E.H. 1983, A\&AS, 54, 55.

Peimbert, M. 1987, in Star Forming Regions, eds. M. Peimbert \& J. Jugaku (Dordrecht, Reidel), p.111. 
Pilachowski, C., Hobbs, L.M., De Young, D.S. 1989, ApJ, 345, L39.

Pinsonneault, M.H., Deliyannis, C.P., Demarque, P. 1992, ApJS, 78, 179.

Rebolo, R., Molaro, P., Abia, C., Beckman, J.E. 1988, A\&A, 193, 193.

Ryan, S.G., Norris, J.E., Bessell, M.S., Deliyannis, C.P. 1992., ApJ, 388, 184.

Schatzman, E. 1991, Mem.S.A.It., 62, 111.

Smith, M.A., Karp, A.H. 1978, ApJ, 219, 522.

Smith, M.A., Karp, A.H. 1979, ApJ, 230, 156.

Smith, V.V., Lambert, D.L., Nissen, P.E. 1992 (in preparation).

Sneden, C., Kraft, R.P., Prosser, C.F., Langer, G.E. 1991, AJ, 102, 2001.

Soderblom, D.R. 1991, Mem.S.A.It., 62, 43.

Spiesman, W.J., Wallerstein, G. 1991, $A J, 102,1790$.

Spite, M., Spite, F. 1991, $A \& A, 252,689$.

Spite, F. 1991, Mem.S.A.It., 62, 11.

Tomkin, J., Lemke, M., Lambert, D.L., Sneden, C. 1992 (preprint).

VandenBerg, D.A. 1992. ApJ, 391, 685. 